\title{
Control de la variabilidad en la programación de pacientes electivos en salas de cirugía*
}

\section{Variability Control in the Scheduling of Elective Patients in Operating Rooms}

\section{Controle da variabilidade no agendamento de pacientes eletivos em salas de cirurgia}

Fecha de recepción: 29-11-14 Fecha de aceptación: 06-03-15 Disponible en línea: 30-06-15 doi: 10.11144/Javeriana.rgyps18-28.cvpp

Como citar este artículo:

Díaz-López LP, Fuquen-Fraile L, Barrera D, González-Neira EM, García-Herreros LG, Suárez DR. Control de la variabilidad en la programación de pacientes electivos en salas de cirugía. Rev. Gerenc. Polít. Salud. 2015; 14(28): 78-87. http://dx.doi.org/10.11144/Javeriana.rgyps18-28.cvpp

Laura P. Díaz-López**

Leide Fuquen-Fraile***

David Barrera****

Eliana M. González-Neira*****

Luis G. García-Herreros $* * * * * *$

Daniel R. Suárez $* * * * * *$

Artículo de investigación. Proyecto: Impacto al implementar estrategias de ordenamiento en la programación de cirugías, junio del 2014-en ejecución. Entidad financiadora: Pontificia Universidad Javeriana.

** Asistente de Investigación del Departamento de Ingeniería Industrial de la Pontificia Universidad Javeriana. Correo electrónico:1diaz@javeriana.edu.co.

**** Ingeniera industrial, directora de Salas de Cirugía de la Fundación Santa Fe de Bogotá. Correo electrónico: leide. fuquen@fsfb.org.co.

***** Ing. MSc., profesor asistente del Departamento de Ingeniería Industrial de la Pontificia Universidad Javeriana. Correo electrónico: barrera-o@javeriana.edu.co.

****** Ing. MSc., profesor asistente del Departamento de Ingeniería Industrial de la Pontificia Universidad Javeriana. Correo electrónico: eliana.gonzalez@javeriana.edu.co.

******** MD, cirujano de tórax, director del Departamento de Cirugía de la Fundación Santa Fe de Bogotá. Correo electrónico:

78 luis.garcia@fsfb.org.co. Correo electrónico: d-suarez@javeriana.edu.co. Dirección: Cra. 7a No. 40-62 - Ed. Maldonado, Piso 3. Bogotá, Colombia. 


\section{Resumen}

El Departamento de Cirugía genera una buena parte de los ingresos de un hospital. Este estudio evalúa la variabilidad en la programación de las cirugías y cómo esta atenta contra la eficiencia del servicio. Este estudio implementó un algoritmo para la generación de dos programaciones que luego fueron comparadas con la realizada manualmente en un hospital en Colombia. Las dos programaciones generadas se diferencian entre sí al incluir o no las máximas desviaciones en los tiempos de duración de las cirugías, asumiendo que estas son causadas por factores externas al proceso y pueden ser eliminadas. Los resultados muestran que al emplear un algoritmo automático para programar las cirugías, podrían mejorarse el tiempo de respuesta y la utilización de las salas. Aún más, se pudo concluir que la variabilidad asociada a la programación manual tiene un mayor impacto que la asociada a otros factores en los indicadores de desempeño del servicio.

Palabras clave: admisión y programación de personal; quirófanos; investigación operativa; servicio de cirugía en hospital; eficiencia organizacional; listas de espera

\section{Abstract}

The Surgery Department generates a sizeable amount of the income of a hospital. This study evaluates the variability in the scheduling of surgeries and how this threatens the efficiency of the service. This study uses an algorithm for the generation of two schedules that were later compared with a manually-generated schedule in a hospital in Colombia. The difference between the generated schedules is the inclusion or lack thereof of the maximum time deviations in surgery times, under the assumption that these are caused by external factors and may be eliminated. Results show that by using an automatic algorithm to schedule the surgeries response time and operating room use can be improved. Moreover, it can be concluded that the variability associated with manual scheduling has a greater impact than the one associated to other factors in the service performance indicators.

Keywords: admission and personnel scheduling; operative research; hospital surgery service; organizational service; waiting lists

\section{Resumo}

O Departamento de Cirurgia gera uma boa parte das rendas de um hospital. Este estudo avalia a variabilidade no agendamento das cirurgias e como ele atenta contra a eficiência do serviço. Este estudo implementou um algoritmo para a geração de dois agendamentos após comparados com aqueles realizados manualmente em um hospital na Colômbia. Os dois agendamentos gerados diferenciam-se entre se por incluir ou não os máximos afastamentos nos tempos de duração da cirurgia, assumindo que estes são causados por fatores externos ao processo e podem ser eliminados. Os resultados mostram que usando um algoritmo automático para programar cirurgias, poderia se melhorar o tempo de resposta e a utilização das salas. Além disso, pode-se concluir que a variabilidade associada ao agendamento manual tem maior impacto que a associada a outros fatores nos indicadores de desempenho do serviço.

Palavras-chave: admissão e agendamento de pessoal; quirófanos; pesquisa operativa; serviço de cirurgia em hospital; eficiência organizacional; listas de espera 


\section{Introducción}

Los prestadores de servicios de salud a nivel mundial se vienen enfrentando a múltiples desafíos en los últimos años, como por ejemplo el incremento y envejecimiento de la población, la aparición de nuevas enfermedades y las continuas restricciones presupuestales (1). Lo anterior ha implicado para las entidades, el reto de encontrar un balance entre la calidad del servicio y la eficiencia del sistema (2). Particularmente, los hospitales buscan una mejora continua en la operación del servicio de cirugía, debido a que los ingresos allí generados corresponden a alrededor del $40 \%$ de los ingresos totales del hospital (3). Adicionalmente, en el servicio de cirugía se concentra la mayor inversión en tecnología, se atienden los pacientes de más alto riesgo y las expectativas de buenos resultados son más evidentes (4). No obstante, comúnmente se reportan problemas en las salas de cirugía que afectan su productividad. Los problemas más comunes incluyen que las cirugías no empiezan a tiempo ni se tardan lo planeado, los insumos y los equipos no están siempre disponibles y el tiempo entre cirugías no es consistentemente corto (4). Todos estos factores afectan el desempeño de un departamento de cirugías, evaluado en términos del porcentaje de utilización y el tiempo de oportunidad, entre otros aspectos.

Diversos autores han identificado la gran variabilidad en el servicio de cirugía como una característica que impide lograr un mejor servicio (5). Esta variabilidad tiene múltiples orígenes, por ejemplo las condiciones propias de los pacientes, la competencia del personal médico, las políticas organizacionales, etc. Muchas de estas fuentes de variabilidad son intrínsecas al servicio de cirugía, como lo es la condición médica de los pacientes; por tanto, la variabilidad introducida es manejable pero imposible de eliminar. Este tipo de variabilidad es llamada natural (6). Por otro lado, otras fuentes de variabilidad son extrínsecas al servicio de cirugía y la variabilidad que introducen es llamada artificial. Esta última debe ser identificada y eliminada para lograr un servicio eficiente (6). Al gestionar apropiadamente la variabilidad del proceso se ha llegado a reducir el número de horas extras y aumentar la utilización y el volumen de pacientes $(7,8)$.

Trabajos anteriores han identificado la programación de las cirugías como la mayor fuente de variabilidad del servicio $(7,9,10)$. Regionalmente, se han realizado unas pocas investigaciones encaminadas a la optimización del servicio de cirugía y, particularmente, a las estrategias de programación de los quirófanos. Velasco et al. (11) concluyen que en Colombia, el uso de metodologías formales para soportar la toma de decisiones en el sector salud necesita lograr una participación activa de los hospitales, a fin de aumentar la probabilidad de éxito en la implementación. Aún más, una revisión de literatura reciente revela tan solo un estudio latinoamericano entre ochenta trabajos citados en el área de interés (12). Finalmente, una encuesta piloto, parte del presente estudio, encontró que los tres hospitales consultados, de alta complejidad (nivel IV) y ubicados en la ciudad de Bogotá (Colombia), realizan la programación de cirugías de forma manual, siendo propensa a una alta variabilidad que podría afectar la eficiencia del servicio.

Este estudio tiene como objetivo 1) evaluar la variabilidad del servicio (en función del número diario de horas de cirugía y, especialmente, la programación de las cirugías) y 2) demostrar la conveniencia de una programación realizada con un algoritmo que reduzca la variabilidad, al eliminar las consideraciones subjetivas al momento de programar las cirugías. Estos beneficios podrían repercutir positivamente en indicadores estratégicos de la organización, como lo son el tiempo de oportunidad y la 
ocupación del servicio $(7,8)$. Este proyecto se realizó con el apoyo de un hospital de alta complejidad, a través del suministro de información relevante para caracterizar y evaluar el desempeño actual de sus salas de cirugía y su sistema de programación.

\section{Metodología}

Para caracterizar el nivel de variabilidad dentro del servicio y evaluar la conveniencia de una programación automática de cirugía, se empleó una metodología con tres etapas: descripción y diagnóstico del servicio, implementación y uso de algoritmo para programación automática y evaluación de la variabilidad e impacto de la programación automática. En breve, este estudio experimental emplea un registro de cirugías reales para medir el posible impacto de utilizar un algoritmo automático para crear la programación del servicio. El posible impacto es evaluado comparando indicadores de desempeño de la programación real del servicio con los que se hubieran obtenido al usar la programación automática.

\section{Fase 1. Descripción y diagnóstico del servicio}

Del registro del servicio de cirugía seleccionado se extrajo información de especialidad, sala, hora de inicio y hora de finalización para todas las cirugías, durante un periodo de 31 días. Este periodo fue definido como de actividad regular o promedio por la dirección del servicio. En total se registraron 655 cirugías de veinticinco especialidades, realizadas en diez salas. El servicio de cirugía seleccionado emplea una programación por bloques, de acuerdo a la demanda de las especialidades; esto es, define unos horarios en los que determinadas salas son de uso exclusivo para una determinada especialidad. La definición de los bloques (especialidades y extensión de los bloques) fue verificada con los datos de demanda para corroborar su conveniencia; sin embargo, este estudio no identificó la necesidad de modificarlos y se tomaron como parte de las características propias del servicio.

El desempeño de las salas fue medido en términos de dos indicadores: porcentaje de utilización (ecuación 1) y longitud promedio de las franjas ociosas (ecuación 2). En general, se espera que un servicio de cirugías eficiente tenga porcentajes de utilización cercanos al $85 \%$ (13). Asimismo, es deseable que las franjas ociosas estén concentradas; esto es, que tengan la mayor duración posible, para poder ocuparlas con nuevos procedimientos.

Porcentaje de utilización $=\left(\frac{\text { minutos utilizados }}{\text { minutos disponibles }}\right) * 100$

Longitud promedio de franjas ociosas $=\frac{\text { minutos ociosos }}{\text { número de franjas ociosas }}$

Para evaluar la variabilidad en el servicio se comparó el tiempo diario de cirugías programadas y no programadas. Se espera que la variabilidad para cirugías programadas sea menor que la encontrada en cirugías no programadas (e.g., pacientes que entran por urgencias). La variabilidad se estimó con el coeficiente de variación del tiempo diario de cirugía.

\section{Fase 2. Algoritmo para programación au- tomática}

Se propone el uso de un algoritmo para realizar la programación semanal del servicio de cirugía. El objetivo es eliminar la variabilidad explicada por las características propias de una programación manual, propensa al error y subjetiva al programador. El algoritmo desarrollado utiliza una regla de despacho Worst Fit, en un escenario determinístico. Este enfoque fue seleccionado por dos razones: a) es simple, flexible y se ajusta a las 
características propias del servicio de cirugía del estudio, y b) esta regla de despacho ha sido aplicada exitosamente en servicios de salud similares (10).

Los parámetros considerados en el algoritmo incluyen: a) el número de la orden de la cirugía, b) la duración de la cirugía, c) la especialidad, d) la cantidad de salas disponibles, e) el número de días a programar, y f) la disposición de bloques en la programación (salas y horarios reservados por especialidad debido a restricciones en personal, equipos, etc.).

Para programar una cirugía, el algoritmo verifica si pertenece a una especialidad que tiene reservado un bloque. En caso de ser así, programa la cirugía en el bloque de la especialidad correspondiente que tenga menor ocupación, de lo contrario, busca en los bloques libres y asigna la cirugía en el que disponga de más espacio disponible. Cuando no hay espacio disponible para asignar una cirugía en la semana, se incluye en la lista de “cirugías no asignadas". Dentro de esta lista se pueden encontrar cirugías que no fueron asignadas porque la duración promedio es superior a la del turno de trabajo más largo en el servicio (siete horas en el caso estudiado), y estas deben asignarse manualmente, de acuerdo al espacio disponible que haya quedado tras programar las cirugías de la semana o priorizar en la programación de una semana siguiente. En la figura 1 se presenta un esquema general del funcionamiento del algoritmo de programación.

Fase 3. Evaluación de la variabilidad inducida por la programación manual

Finalmente, para hacer una evaluación del impacto que tiene la programación manual, se efectuaron experimentos computacionales y pruebas estadísticas, considerando tres escenarios:

- Escenario 1: la programación y la duración de las cirugías se mantienen como

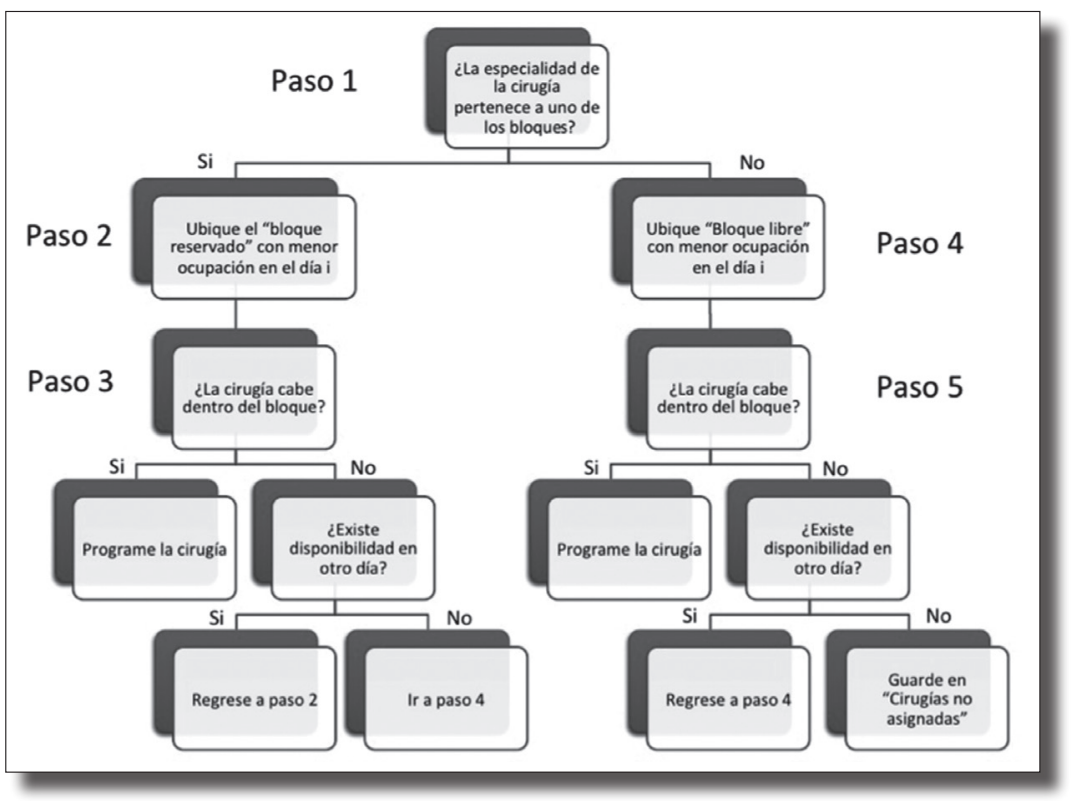

Figura 1. Algoritmo de programación empleado una regla de despacho Worst Fit. 
están en la base de datos del hospital. En este escenario hay variabilidad artificial, producida por la programación manual, y variabilidad natural del proceso.

- $\quad$ Escenario 2: la duración de las cirugías se mantiene y la programación se hace con el algoritmo diseñado. De esta manera, se elimina la variabilidad introducida por la programación manual.

- Escenario 3: la programación de las cirugías se hace con el algoritmo y cambian las duraciones de las cirugías inusualmente largas. En este escenario a todas las cirugías cuya duración está por fuera de límites de control definidos por subespecialidad (i.e., tres desviaciones estándar) se les asigna un nuevo tiempo, igual al promedio de la especialidad. De esta manera, se asume que se elimina toda la variabilidad artificial.

Se realizaron comparaciones entre el escenario 1 y los escenarios 2 y 3 , en términos del porcentaje de utilización y la duración media de las franjas ociosas. Adicionalmente, se midió el cambio en el tiempo de oportunidad para las cirugías (ecuación 3).

$$
\Delta T O=\text { Hora }_{(\text {Escenario 1) }}-\text { Hora }_{\text {propuesta }}
$$

Se realizaron pruebas de hipótesis para evaluar si el cambio en el tiempo de oportunidad $(\Delta T O)$ entre las programaciones es positivo (escenario $1 v s$. escenario 2 y escenario $1 v s$. escenario 3), lo cual significaría una reducción en el tiempo de oportunidad al implementar el algoritmo de programación propuesto.

Para evaluar la significancia del posible cambio en el tiempo de oportunidad y la contribución de cada especialidad en el cambio del tiempo de oportunidad, se realizaron una prueba de hipótesis y un análisis de varianza (Anova) de dos factores (escenario y espe- cialidad médico-quirúrgica). Posteriormente, se realizó un análisis de diferencia mínima significativa (DMS), a fin de determinar qué especialidades eran las responsables del cambio significativo, si lo había, entre los diferentes tipos de programación (i. e., escenarios).

\section{Resultados}

\section{Indicadores de desempeño del servicio}

Para caracterizar el funcionamiento del departamento de cirugía se calcularon los indicadores: porcentaje de utilización y longitud promedio de franjas ociosas. Con un nivel de confianza del $95 \%$, el porcentaje de utilización promedio semanal de todo el servicio de cirugías estuvo en $60,3 \% \pm 7,2 \%$, mientras que al separar el indicador por sala se observan comportamientos diferentes. Por ejemplo, la sala con mayor ocupación es multifuncional y con gran capacidad para equipos e instrumental, por esta razón, es la más solicitada por los médicos $(84,4 \% \pm$ $17,8 \%$ ). Por otra parte, la longitud promedio de franjas ociosas estuvo entre 2,4 y 3,3 horas de lunes a viernes y alcanzó las 7,0 horas los sábados. En general, la capacidad instalada puede permitir la realización de un mayor número de cirugías, sin embargo, las franjas libres no son en promedio lo suficientemente largas como para incluir cirugías adicionales.

La variabilidad del servicio se evidenció en el flujo de pacientes a través de este. El flujo de pacientes programados, en términos del tiempo utilizado por día, fue tan variable como el flujo de pacientes no programados, lo que indicaría una alta variabilidad (ver figura 2). Esta variabilidad se cuantificó empleando el coeficiente de variación de ambos flujos y que resultó del mismo orden de magnitud para casos programados y para casos no programados: 0,61 vs. 0,68 . 


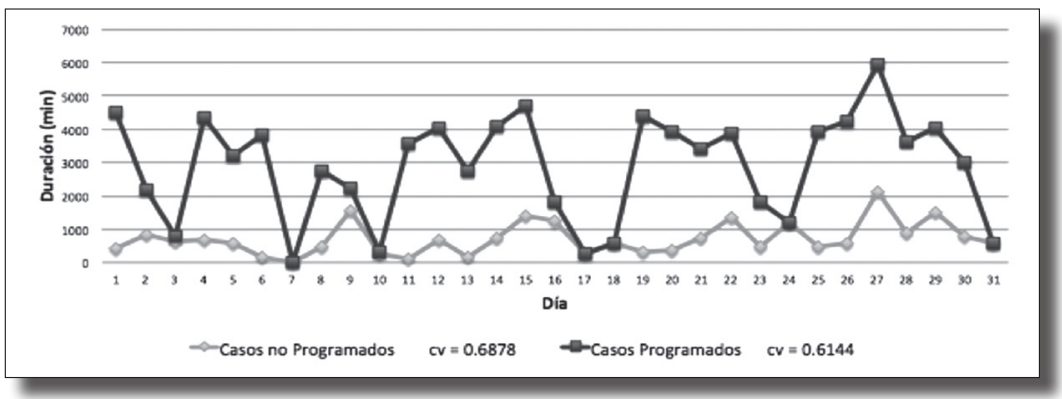

Figura 2. Tiempo de CIRUgía POR día y COEFICIENTE de VARIACión PARA CASOS PROGRAMAdos y No PROGRAMAdos DURANTE LA DURACIÓN DEL ESTUDIO

Fuente: elaboración propia

Por medio de gráficas de control de la duración de las cirugías por especialidad, se identificaron dieciséis casos inusualmente largos durante el tiempo de observación. Se asumió que la duración inusual de estas cirugías no se debía a factores propios del servicio de cirugía y que, por lo tanto, era un reflejo de una variabilidad artificial que podría ser corregida o eliminada en un futuro. El escenario de programación sin variabilidad artificial (escenario 3), que a continuación es empleado para evaluar el algoritmo de programación propuesto, es definido mediante la modificación de la duración de estas cirugías con duración inusual a una duración promedio para la subespecialidad a la cual pertenecen.

\section{Evaluación del algoritmo propuesto y de la} variabilidad introducida por la programación manual

Con el fin de evaluar el impacto del algoritmo propuesto y la variabilidad en la programación, se ejecutó el algoritmo de programación automática con una regla de despacho para crear los escenarios 2 y 3 , para luego compararlos con el escenario 1. Las comparaciones fueron realizadas por medio del porcentaje de utilización, la longitud promedio de franjas ociosas y el cambio en el tiempo de oportunidad $(\triangle T O)$. Posteriormente, se utilizaron pruebas de hipótesis y un análisis de varianza (Anova) para comprobar la validez de los resultados.
Con el fin de utilizar estas pruebas estadísticas se comprobó, en primera instancia, que las poblaciones de indicadores se distribuyen normalmente (valor-p > 0,150) y que sus varianzas son homogéneas (valor-p $=0,185$ ).

El porcentaje de utilización presentó una diferencia mínima entre el escenario 1 y los escenarios 2 y 3 . En el escenario 2 esa diferencia se debió a la no inclusión de quince cirugías con una duración mayor a siete horas (i. e., duración máxima de un turno del personal) y que deben ser programadas manualmente en las franjas ociosas disponibles. En el escenario 3 la diferencia en la utilización fue aún menor, debido a tan solo siete cirugías que se dejaron de programar.

Con la longitud promedio de franjas ociosas se evaluó el nivel de dispersión de las cirugías en la programación, teniendo en cuenta la sala y el día en que se realizó cada una. En la tabla 1 se observan las franjas no asignadas para la programación actual y las programaciones automáticas de los escenarios 2 y 3 . Estas últimas programaciones, al aumentar el porcentaje de utilización en los primeros días de la semana, permitieron obtener franjas libres promedio lo suficientemente largas (más de 6,6 horas) como para poder programar posibles cirugías adicionales de miércoles a sábado. Estas cirugías adicionales podrían ser tanto casos de urgencias 
Tabla 1. DuRación O LONGITUd PROMEdIO de FRANJAS OCIOSAS PROMEDIO DE LA PROGRAMACIÓN NO ASIGNADAS A CIRUGías

\begin{tabular}{|c|c|c|c|c|c|c|}
\hline Escenario & Lunes & Martes & Miércoles & Jueves & Viernes & Sábado \\
\hline 1 & 2,9 & 3,3 & 2,4 & 2,6 & 3,3 & 7,0 \\
\hline 2 & 1,7 & 2,0 & 6,6 & 8,6 & 10,3 & 12,5 \\
\hline 3 & 0,5 & 2,0 & 6,6 & 8,1 & 10,1 & 12,5 \\
\hline
\end{tabular}

Fuente: elaboración propia

como cirugías inicialmente en lista de espera o programadas para una semana posterior.

Con independencia del escenario, el $\triangle T O$ por especialidad para las cirugías más frecuentes resultó siempre positivo cuando se empleó una programación automática (ver tabla 2), por lo que se evidencia una tendencia a programar cirugías en una fecha anterior a la que en realidad las cirugías se realizaron. Esta tendencia fue evaluada por una prueba hipótesis (Ho: $\Delta T O=0$, Ha: $\Delta T O>0$ ) que permitió determinar si hubo una reducción significativa en el tiempo de programación de las cirugías. Como resultado de la prueba de hipótesis se obtuvo que tanto el escenario 2 como el escenario 3 redujeron significativamente el tiempo de oportunidad con respecto al escenario 1 (de programación manual), con valores-p menor a 0,0001 e igual a 0,0035 , respectivamente.

El Anova de dos factores (escenarios, especialidades) determinó que sí existe un efecto significativo de estos factores o de su interacción en el $\triangle T O$. Con un valor-p $<0,001$ se determinó que sí hay diferencias significativas en el $\triangle T O$ ocasionadas por las especialidades, mientras que con un valor-p igual a 0,682 se concluye que no hay diferencias significativas en el $\triangle T O$ entre los escenarios 2 y 3 . Esto último indica que la mejora del servicio debe ser primordialmente realizada a través de una mejora del método de programación de las cirugías, pues la eliminación de la variabilidad generada por problemas de otro tipo, por ejemplo organizacionales, no representó un cambio significativo.

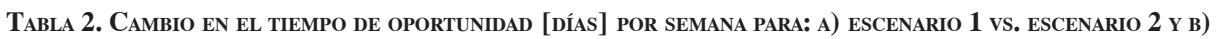
ESCENARIO 1 VS. ESCENARIO 3. LAS DIFERENCIAS POSITIVAS INDICAN UN ADELANTO EN LA FECHA DE LA CIRUGía.

\begin{tabular}{|l|c|c|c|c|c|c|c|c|}
\hline \multirow{2}{*}{ Especialidad } & \multicolumn{3}{|c|}{ Escenario 2 vs. escenario 1 } & \multicolumn{3}{c|}{ Escenario 3 vs. escenario 1 } \\
\cline { 2 - 11 } & \multicolumn{4}{|c|}{ Semana } & \multicolumn{4}{c|}{ Semana } \\
\cline { 2 - 11 } & $\mathbf{1}$ & $\mathbf{2}$ & $\mathbf{3}$ & $\mathbf{4}$ & $\mathbf{1}$ & $\mathbf{2}$ & $\mathbf{3}$ & $\mathbf{4}$ \\
\hline Anestesiología & 0,44 & 0,47 & 0,18 & 0,11 & 0,22 & 0,58 & 0,18 & 0,11 \\
\hline Cirugía General & 0,82 & 1,06 & 1,17 & 0,14 & 0,76 & 1,44 & 1,21 & 0,43 \\
\hline Cirugía Plástica & 2,00 & 1,74 & 1,57 & 1,06 & 1,74 & 1,79 & 1,57 & 1,06 \\
\hline Ortopedia & 1,23 & 0,03 & 1,09 & $-0,02$ & 1,19 & 0,03 & 1,19 & 0,12 \\
\hline Otorrinolaringología & 1,50 & 0,69 & 2,60 & 0,88 & 0,13 & 0,31 & 2,60 & 0,88 \\
\hline Urología & 2,09 & 1,88 & 1,64 & 1,55 & 2,09 & 2,00 & 1,64 & 1,55 \\
\hline Gineco-obstetricia & 0,50 & $-0,60$ & 2,00 & $-0,70$ & 0,00 & $-0,60$ & 2,00 & $-0,60$ \\
\hline Total por servicio & 1,23 & 0,75 & 1,46 & 0,43 & 0,88 & 0,79 & 1,48 & 0,50 \\
\hline
\end{tabular}

Fuente: elaboración propia. 
Finalmente, la prueba de DMs determinó que en el servicio de cirugía estudiado, las especialidades de Urología, Cirugía Plástica, Otorrinolaringología y Cirugía General tienen un $\triangle T O$ significativamente mayor que otras especialidades (valores-p $<0,035$ ), por lo que fueron las especialidades que más se beneficiaron del algoritmo de programación automática.

\section{Discusión y conclusiones}

El impacto del uso de herramientas cuantitativas en la programación de cirugías ha sido medido en diferentes contextos, restricciones y tamaños de muestra (12). Por ejemplo, Xiang et al. (14) midieron la efectividad de un algoritmo de optimización en la programación de diez salas de cirugías, a partir de una muestra de diez días de trabajo y alrededor de 450 cirugías. En este estudio, al igual que lo propuesto en Saadouli et al. (15), se tomó una muestra de 31 días de trabajo del servicio de cirugía de un hospital privado, compuesto por diez salas para procedimientos electivos y una sala dedicada a la atención de urgencias.

Con el fin de diagnosticar el funcionamiento operativo de las salas de cirugía, se evaluaron los indicadores de desempeño porcentaje de utilización y longitud promedio de franjas ociosas. Esta evaluación permitió establecer que la capacidad instalada permite la realización de un mayor número de cirugías, sin embargo, las franjas libres dadas por la programación manual actual no son en promedio lo bastante largas como para incluir cirugías adicionales. Se evidenció también la existencia de variabilidad artificial en la programación manual, al encontrarse que el coeficiente de variación del tiempo diario empleado en cirugías es similar para los casos programados y para los no programados.

86 Para reducir la variabilidad asociada a la programación se implementó un algoritmo que aumentó la ocupación programada del servicio en los primeros días de la semana y liberó franjas en la programación al final de la misma semana, lo bastante grandes como para programar cirugías adicionales a las inicialmente consideradas. Adicionalmente, el algoritmo de programación también propició un cambio favorable en el tiempo de oportunidad $(\triangle T O)$, haciendo posible asignar las cirugías en promedio un día antes de lo asignado mediante la programación manual.

Finalmente, se comprobó también que la variabilidad existente en el servicio se debe en mayor medida al proceso de programación de las cirugías, lo cual es coherente con lo presentado por Litvak (7). La verificación de esa afirmación se dio al encontrar que el cambio en el tiempo de oportunidad $(\triangle T O)$ entre los escenarios 2 y 3 (con y sin variabilidad artificial debido a causas diferentes a la causada por una programación manual) no es significativo, pero sí lo fue para el cambio entre la programación manual y la programación automática.

Es difícil hacer una comparación directa con estudios previos, debido a que los datos de las cirugías son propios del hospital donde se ha realizado el estudio, y no necesariamente deben ser similares a las cirugías realizadas en otros hospitales. Sin embargo, los resultados presentados son coherentes con los reportados anteriormente, al reducir la variabilidad del servicio de cirugías como el aumento de la utilización y mejores tiempos de oportunidad, entre otras ventajas $(7,8)$.

Otros estudios también han determinado la programación de las cirugías (12), bien empleando metodologías de optimización determinísticas $(1,16)$, o bien valiéndose de metodologías estocásticas $(3,17)$. Muchos de estos trabajos son bastante elaborados y cercanos a la realidad, al tener en cuenta la incertidumbre de diversos factores y diferen- 
tes variables de decisión (ver, por ejemplo, Pérez- Gladish et al. [18]). Sin embargo, esos estudios son difíciles de generalizar e implementar, y aun con su complejidad, no tienen en cuenta la totalidad de los factores y las variables del problema. El método de programación presentado en este estudio, por el contrario, es fácil de implementar y conlleva ventajas significativas con respecto a la forma de generar la programación del servicio de cirugía que se realiza actualmente en los hospitales regionales.

Este estudio evaluó la variabilidad del servicio de cirugías de un hospital de alta complejidad en Bogotá y demostró la conveniencia de una programación automática, al eliminar la variabilidad asociada a la programación manual en términos de utilización, ocupación y tiempo de oportunidad en el servicio de cirugía. Futuros estudios deben incluir la implementación de los algoritmos de programación automática en clínica y en la medición de su impacto, empleando índices comúnmente utilizados como la ocupación del servicio, horas extras, disponibilidad para casos emergentes o urgentes y número de cambios en la programación diaria.

\section{Referencias bibliográficas}

1. Fei H, Chu C, Meskens N. Solving a Tactical Operating Room Planning Problem by a Column-GenerationBased Heuristic Procedure with Four Criteria. Annals of Operations Research. 2009; 166 (1): 91-108.

2. Brailsford S, Vissers J. OR in Healthcare: A European Perspective. Eur J Oper Res. 2011; 212 (2): 223-34.

3. Denton B, Viapiano J, Vogl A. Optimization of Surgery Sequencing and Scheduling Decisions under Uncertainty. Health Care Manag Sci. 2007; 10 (1): 13-24.

4. Kaye AD, Fox C, Fox III CJ, Urman RD. Operating Room Leadership and Management. Cambridge University Press; 2012.

5. Litvak E, Long MC. Cost and Quality under Managed Care: Irreconcilable Differences. Am J Manag Care. 2000; 6 (3): 305-12.

6. Litvak E, Long M, Prenney B, Fuda K, LevtzionKorach O, McGlinchey P. Improving Patient Flow and Throughput in California Hospitals Operating
Room Services. Guidance Document Prepared for the California Healthcare Foundation ( $\mathrm{CHCF}) ; 2007$.

7. Litvak E. Optimizing Patient Flow by Managing its Variability. Front Office to Front Line: Essential Issues for Health Care Leaders. Oakbrook Terrace, IL, Estados Unidos: Joint Commission Resources; 2005. pp. 91-111.

8. Smith CD, Spackman T, Brommer K, Stewart MW, Vizzini M, Frye J, et al. Re-engineering the Operating Room Using Variability Methodology to Improve Health Care Value. J Am Coll Surg. 2013; 216 (4): 559-68.

9. McManus ML, Long MC, Cooper A, Litvak E. Queuing Theory Accurately Models the Need for Critical Care Resources. Anesthesiology. 2004; 100 (5): 1271-6.

10. Dexter F, Macario A, Traub RD. Which Algorithm for Scheduling Add-on Elective Cases Maximizes Operating Room Utilization?: Use of Bin Packing Algorithms and Fuzzy Constraints in Operating Room Management. Anesthesiology. 1999; 91 (5): 1491-500.

11. Velasco N, Barrera D, Amaya CA. Logística hospitalaria: Lecciones y retos para Colombia. En: Bernal O, Gutiérrez C, editores. La salud en Colombia. Logros, retos y recomendaciones. Bogotá: Uniandes; 2012. pp. 309-43.

12. Velásquez-Restrepo PA, Rodríguez-Quintero $\mathrm{AK}$, Jaén-Posada JS. Aproximación metodológica a la planificación ya la programación de las salas de cirugía: una revisión de la literatura. Gerencia y Políticas de Salud. 2013; 12 (24): 249-66.

13. Long MC. Re-engineering the OR. En: Litvak E, Long M, Prenney B, Fuda K, Levtzion-Korach O, McGlinchey P, editors. Improving Patient Flow and Throughput in California Hospitals Operating Room Services. Boston, Estados Unidos: Boston University Health Policy Institute; 2007. pp. 65-110.

14. Xiang W, Yin J, Lim G. A Short-Term Operating Room Surgery Scheduling Problem Integrating Multiple Nurses Roster Constraints. Artif Intell Med. 2014. doi: 10.1016/j.artmed.2014.12.005

15. Saadouli H, Jerbi B, Dammak A, Masmoudi L, Bouaziz A. A Stochastic Optimization and Simulation Approach for Scheduling Operating Rooms and Recovery Beds in an Orthopedic Surgery Department. Comput Ind Eng. 2015; 80: 72-9.

16. Kuo PC, Schroeder RA, Mahaffey S, Bollinger RR. Optimization of Operating Room Allocation Using Linear Programming Techniques. J Am Coll Surg. 2003; 197 (6): 889-95.

17. Lamiri M, Grimaud F, Xie X. Optimization Methods for a Stochastic Surgery Planning Problem. Int J Prod Econ. 2009; 120 (2): 400-10.

18. Pérez-Gladish B, Arenas-Parra M, Bilbao-Terol A, Rodríguez-Uría MV. Management of Surgical Waiting Lists through a Possibilistic Linear Multiobjective Programming Problem. Applied Mathematics and Computation. 2005; 167 (1): 477-95. 